Relations industrielles

Industrial Relations

\title{
Les conseils d'arbitrage devraient-ils être abolis?
}

Volume 7, numéro 3, juin 1952

URI : https://id.erudit.org/iderudit/1023052ar

DOI : https://doi.org/10.7202/1023052ar

Aller au sommaire du numéro

\section{Éditeur(s)}

Département des relations industrielles de l’Université Laval

\section{ISSN}

0034-379X (imprimé)

1703-8138 (numérique)

Découvrir la revue

Citer ce document

(1952). Les conseils d'arbitrage devraient-ils être abolis? Relations industrielles / Industrial Relations, 7(3), 193-194. https://doi.org/10.7202/1023052ar
Résumé de l'article

Les conseils d'arbitrage devraient-ils être abolis? Cette intéressante question a été soulevée dans l'éditorial du numéro d'hiver 1952 de la revue " Public Affairs » et présentée de la manière suivante.
Tous droits réservés @ Département des relations industrielles de l’Université Laval, 1952
Ce document est protégé par la loi sur le droit d'auteur. L'utilisation des services d'Érudit (y compris la reproduction) est assujettie à sa politique d'utilisation que vous pouvez consulter en ligne.

https://apropos.erudit.org/fr/usagers/politique-dutilisation/ 


\section{LES CONSEILS D'ARBITRAGE DEVRAIENT-ILS ETRE ABOLIS ?}

\section{Les conseils d'arbitrage devraient-ils être abolis? Cette intéressante question a été soulevée dans l'éditorial du numéro d'hiver 1952 de la revue «Public Affairs»1 et présentée de la manière suivante.}

«Le conseil d'arbitrage remonte à 1907 et est accepté comme critère par la législation canadienne fédérale et provinciale en matière de relations industrielles. Mais les gens qui doivent faire fonctionner ce système et qui en dépendent ne veulent plus de toute façon l'accepter d'emblée. Pourquoi?

Tout d'abord, les délais prévus par la loi dans les différentes étapes pour arriver à une solution, ou à une impasse, à compter de la reconnaissance de l'union, à la négociation, à la conciliation, compte tenu des audiences du conseil d'arbitrage, les rapports, «la période de refroidissement» (cooling off period) [laquelle, si elle se prolonge indûment, peut devenir une période d'échauffement (hotting up period) ]. Ces tactiques de délai souvent employées comme excellent moyen de tuer les unions faibles constituent également une arme à laquelle peuvent recourir les employeurs déterminés à rester maîtres de tout leur domaine; elles peuvent aussi être utilisées contre les unions vraiment puissantes pour différer toute décision jusqu'au milieu de l'hiver, ou jusqu'à une saison-morte au moment où leur pouvoir de négocier est sérieusement affaibli.

Dans l'ensemble, les conseils d'arbitrage sont déplorables pour les unions. Mais quel est leur effet sur les employeurs? sur le public? L'employeur, tout particulièrement le petit employeur dans une industrie concurrentielle peut être pratiquement désemparé par l'incertitude de savoir s'il pourra faire fonctionner son entreprise et dans telle éventualité, quel sera le niveau de la rémunération de la main-d'oeuvre.

Mais l'intérèt public prime sur le bien particulier des travailleurs ou des employeurs, et les conseils d'arbitrage peuvent empêcher les grèves; mais le font-ils en fait?... Ils peuvent les faire différer mais seulement au risque de les rendre alors plus dommageables,... les grèves inopinées mettent la loi en brèche. Cet état de chose est-il dans l'intérêt public?

Après cette argumentation contre les conseils d'arbitrage l'éditorial apporte de nouvelles idées qui s'ajoutent à leur dossier déjà chargé: les conseils d'arbitrage émoussent le sens de responsabilité chez les deux parties, minant ainsi la négociation collective et tendent à confier l'ajustement des griefs à ceux-là mêmes qui en connaissent le moins sur les industries concernées.

Les choses se passent à peu près comme ceci au premier stage des négociations; point n'est besoin de porter attention aux réalités de la situation. \& Plus le point de départ sera élevé, plus haut sera le point d'arrivée; le ciel est la limite. Pendant ce temps, nous aurons eu l'occasion de démontrer à nos membres (ou directeurs) exactement' quels négociateurs tenaces et militants nous sonmes. Si le règlement semble un terrible échec nous pouvons fairc rcjeter le blâme sur le tribunal. Il est beaucoup plus agréable d'agir ainsi plutôt que de commencer par des offres raisonnablement acceptables, de négocier réellement et de porter ensuite le fardeau du blâme pour tout résultat ne satisfaisant pas nos membres.

Alors chaque partie dépose bruyamment sur la table son réquisitoire. Chacune dit «Non» aux propositions de l'autre, de façon dramatique et souvent même de façon flamboyante; chaque groupe de négociateurs proclame à ses mandataires et peut-être au public également sa détermination ferme de résister jusqu'au dernier souffle aux violentes demandes de l'autre, et d'insister sur ses propositions inattaquablement justes, raisonnables et modérées.

(1) Revue nationale trimestrielle dı Canada publiée par The Institute of Public affairs, Université Dalhousì. Halifax, Canada. 
A ce moment, le ministre délègue un officier de conciliation. Aucun résultat. Alors, il nomme d'office un tribunal. Dans la plupart des cas, les deux membres du conseil et le président sont moins renseignés sur l'indus'rie que les parties elles-mêmes. Ils peuvent faire des recommendations tout à fait impraticables: les parties doivent dès ce moment se réunir et essayer d'en venir à un compromis dans une atmosphère déjà empoisonnée par le non sens flagrant qu'ils ont entretenu mutuellement pendant des mois.

L'éditorial conclut de la façon suivante: cette situation devient de plus en plus courante. Cela ne fait de bien à personne. Elle présente un grand danger à une période où les besoins les plus impérieux exigent que chacun comprenne autant que possible quels sont les pôles entre lesquels chaque convention peut être conclue sans effet désastreux sur la société tout entière. Au moment où la responsabilité, la raison, la tolérance et la compréhension sont indispensables à la survie dє la démocratie occidentale, le conseil d'arbitrage nonobstant ses avantages reconnus attribue une prime à l'irresponsabilité, à la déraison, à l'intolérance et à lignorance.

Quelle est donc l'alternative? Laisser les parties agir sans intervention publique d'aucune façon? Non. Il faut pourvoir à la formation d'une assez fort groupe d'experts, d'officiers permanents de conciliation, et laisser le reste à la vraie négociation collective y compris la grève et son cortège, l'autorité publique n'intervenant officiellement que lorsque la santé nationale et la sécurité l'exigent. Cela peut sembler hasardeux, mais au contraire. Les deux parties seront entrainées à agir comme des adultes, et non comme des enfants gâtés. A la longue, les grèves se feront moins nombreuses, les relations industrielles seront meilleures, et la société deviendra plus naturellement démocratique.

\section{DOCUMENTS PONTIFICAUX}

\section{LE CREDIT}

Le 24 octobre 1951, le Pape recevait un groupe de membres du Congrès international du Crédit, à Castel-Gandolfo, et leur adressa la parole en français. Voici le texte de cette allocution, que reproduisait l'Osservatore Romano du 26 octobre 1951.

Votre démarche, à laquelle Nous sommes fort sensible, est, Messieurs, une preuve convaincante de la haute idée que vous avez de votre profession et de votre rôle. Au-dessus d'un intelligent maniement de fonds, au-dessus mème du simple intérêt financier de vos établissements et de leurs clients, vous placez son utilité morale et sociale. Vous marquez la frontière, ou, plus exactement, le carrefour, où se rencontrent le capital, la pensée, le travail.

Alors que des pêcheurs en eau trouble en soulignent, en exagèrent l'opposition, votre tâche consiste à les mettre en relations, à les rendre mutuellement utiles. Surtout sous l'aspect du crédit, qui fait l'objet central de votre Congrès international, vous êtes, bien plus que des exécuteurs ou des mandataires d'opérations bancaires, des conseilleurs.

\section{Saine utilisation des capitaux}

Que de capitaux se perdent dans le gaspillage, dans le luxe, dans l'égoöste et fastidieuse jouissance, ou s'accumulent et dorment sans profit! Il y aura toujours des égoïstes et des jouisseurs, il y aura toujours des avares et des timides à courte vue. Leur nombre pourrait être considérablement réduit, si l'on savait intéresser ceux qui possèdent à un emploi judicieux et profitable de leurs fonds, opulent's ou modestes. C'est, en grande partie, faute de cet intérêt, que l'argent coule ou dort. Pour y remédier, vous pouvez beaucoup par le soin de transformer les simples dépo- 